\title{
Evaluación de la dinámica cardiaca con la aplicación de metodologías basadas en entropía proporcional y la ley Zipf-Mandelbrot
}

\author{
Javier Rodríguez, ${ }^{1}$ Signed Prieto ${ }^{2}$ y Leonardo Ramírez ${ }^{3}$ \\ ${ }^{1}$ Universidad Militar Nueva Granada, Línea de Profundización e Internado Especial: Física y Matemáticas Aplicadas a la Medicina; ${ }^{2}$ Clínica del \\ Country, Centro de Investigaciones; ${ }^{3}$ Universidad Militar Nueva Granada. Bogotá, Colombia
}

\begin{abstract}
Resumen
Introducción: Las metodologías fisicomatemáticas son de utilidad para el diagnóstico de la dinámica cardiaca. Objetivo: Comparar la aplicación de dos métodos matemáticos de evaluación de la dinámica cardiaca. Una basada en proporciones de la entropía y otra en la ley de Zipf-Mandelbrot. Método: Se tomaron 10 registros Holter, cinco de pacientes con enfermedad aguda y cinco normales. Se construyó un atractor numérico, se evaluó la probabilidad, entropía y proporciones de entropía. Para aplicar la segunda metodología se agruparon los valores de frecuencia cardiaca en rangos de 15 latidos/minuto y se aplicó la ley de Zipf-Mandelbrot para obtener la dimensión fractal estadística. Finalmente se comparó la evaluación matemática obtenida por ambas metodologías. Resultados: La metodología basada en las proporciones de la entropía diferenció normalidad, enfermedad y estados intermedios. La segunda metodología diferenció normalıdad de enfermedad aguda mediante el grado de complejidad. Conclusión: Ambas metodologías establecen evaluaciones de ayuda diagnóstica de la dinámica cardiaca de forma objetiva y reproducible. La entropía proporcional permite cuantificar normalidad, enfermedad y evolución entre estados con carácter predictivo y mayor precisión.
\end{abstract}

PALABRAS CLAVE: Frecuencia cardiaca. Entropía. Métodos matemáticos. Dinámica cardiaca.

\begin{abstract}
Introduction: Physical-mathematical methodologies have been useful for the diagnosis of cardiac dynamics. Objective: To compare the application of two mathematical methodologies for cardiac dynamics evaluation, one of them based on entropy proportions and the other based on of Zipf-Mandelbrot law. Method: 10 Holter, 5 acute disease dynamics and 5 normal records were taken. A numerical attractor was constructed; probability, entropy and entropy proportions were evaluated. To apply the second methodology, heart rate values were grouped in 15-beat/min ranges, and Zipf-Mandelbrot's law was applied in order for the statistical fractal dimension to be obtained. Finally, the mathematical evaluation obtained by both methodologies was compared. Results: The methodology based on entropy proportions differentiated normality, disease and intermediate states. The second methodology differentiated normality from acute disease through the degree of complexity. Conclusion: Both methodologies establish diagnostically helpful evaluations of cardiac dynamics in an objective and reproducible way. Proportional entropy allows normality, disease and evolution between states to be quantified in a predictive manner and with higher accuracy.
\end{abstract}

KEY WORDS: Heart rate. Entropy. Mathematics methods. Cardiac dynamics.

Correspondencia:

Javier Rodríguez

E-mail: grupoinsight2025@yahoo.es
Fecha de recepción: 21-12-2016

Fecha de aceptación: 15-01-2018

DOI://dx.doi.org/10.24875/GMM.18003128
Gac Med Mex. 2018;154:287-294

Disponible en PubMed www.gacetamedicademexico.com 


\section{Introducción}

En el estudio de la dinámica cardiaca se han realizado diferentes métodos de evaluación mediante conceptos y teorías físicas y matemáticas entre las que figuran la teoría de sistemas dinámicos, la probabilidad, la entropía y la ley de Zipf -Mandelbrot. La teoría de sistemas dinámicos estudia el estado y la evolución de los sistemas. ' La representación del comportamiento al que tienden los sistemas se realiza mediante atractores caóticos, los cuales se analizan por geometría fractal dada su irregularidad. ${ }^{2}$ El estudio de la dinámica cardiaca a partir de estos métodos ha dado lugar a una neoconcepción de normalidad y enfermedad que contradice la posición convencional de la fisiología basada en la homeostasis. ${ }^{3}$

El lenguaje matemático sustrato de la física moderna está basado en la probabilidad, medición que calcula la posible ocurrencia de determinado evento en el futuro. ${ }^{4}$ La entropía, por su parte, ha sido reinterpretada y aplicada en la mecánica estadística y en la teoría cinética de los gases, entre otros campos..$^{5-7}$ En medicina ha sido empleada para el desarrollo de mediciones como la entropía aproximada y para el estudio de los cambios en la complejidad de los sistemas. ${ }^{8-10} \mathrm{Su}$ aplicabilidad clínica aún está siendo investigada. ${ }^{9}$

Otra metodología matemática es la ley de Zipf, ${ }^{11}$ inicialmente aplicada en lingüística para evaluar la frecuencia de ocurrencia de palabras en un texto; para ello se disponían las frecuencias de palabras de mayor a menor y a cada una se les asignaba un rango, empezando por 1 , que aumentaba a medida que la frecuencia disminuía, lo que evidenciaba un comportamiento hiperbólico. Posteriormente, Mandelbrot desarrolló una linealización y creó el concepto de dimensión fractal estadística para medir el grado de complejidad de los sistemas..$^{12-14}$

La importancia de emplear estas metodologías en ámbitos médicos como la cardiología radica en que las enfermedades de origen cardiovascular están descritas como la principal causa de muerte en el mundo, ${ }^{15}$ por lo que permanentemente se busca el mejoramiento del análisis de la dinámica cardiaca, ${ }^{16-19}$ la cual ha sido analizada a la luz de diversos modelos matemáticos que todavía requieren más estudios que corroboren su aplicabilidad clínica. ${ }^{20,21}$

Goldberger et al. ${ }^{3}$ han realizado una interpretación distinta de normalidad-enfermedad en el marco de la teoría de los sistemas dinámicos, según la cual en la enfermedad predominan dinámicas en extremo aleatorias o muy regulares y en el intermedio se encuentra la normalidad. Se han propuesto nuevos índices para predecir la mortalidad mediante análisis fractales de los intervalos de las razones de riesgo (RR) en pacientes que cursan con infarto agudo de miocardio. ${ }^{17}$ Las investigaciones muestran que el abordaje desde la perspectiva estadística convencional no predice con precisión la variabilidad de la frecuencia cardiaca, por lo cual es menester buscar nuevas opciones y herramientas diagnósticas.

Otra metodología desarrollada en esta misma línea ha permitido diferenciar dinámicas mediante las proporciones de la entropía. ${ }^{22}$ Para confirmar la aplicabilidad clínica de esta metodología se han realizado estudios cegados con 450 y 600 dinámicas cardiacas normales y patológicas; ${ }^{22,23}$ se encontraron valores de sensibilidad y especificidad de $100 \%$ y un coeficiente kappa $=1$. Esta metodología basada en la entropía proporcional también fue aplicada para el estudio de la dinámica cardiovascular en pacientes de una unidad de cuidados intensivos: permitió predecir la evolución a enfermedad aguda, subdiganosticada con parámetros clínicos convencionales. ${ }^{24}$ También ha logrado medir el nivel de gravedad de la dinámica cardiaca en pacientes con diagnóstico clínico de arritmia. ${ }^{25}$

Recientemente se desarrolló la primera metodología de ayuda diagnóstica de la dinámica cardiaca con base en la ley Zipf-Mandelbrot. Las dinámicas normales y agudas fueron caracterizadas matemáticamente mediante el comportamiento hiperbólico de la organización de las frecuencias cardiacas del registro electrocardiográfico correspondiente, lo que mostró que el grado de complejidad de dinámicas sin alteraciones es mayor que el de dinámicas con agudización, con lo que se evaluó el estado de la dinámica cardiaca de una forma simple, cuantificable y reproducible. ${ }^{26} \mathrm{EI}$ propósito de la presente investigación es aplicar esta metodología simultáneamente con otra metodología matemática de ayuda diagnóstica para evidenciar las cuantificaciones logradas con ambas metodologías.

\section{Método}

\section{Definiciones}

- Mapa de retardo: Tipo de atractor que representa la relación entre un punto dado de una serie de tiempo graficado en el eje de las abscisas, y el siguiente punto en la serie de tiempo graficado en el eje de las ordenadas. La serie de tiempo está constituida por las frecuencias cardiacas consecutivas en el tiempo. 
- Rango de pares ordenados de frecuencias cardiacas $(X, Y)$ : Intervalo de frecuencias cardiacas que agrupa todos los pares ordenados cuyas coordenadas se encuentren entre los valores $X$ y $Y$. Los valores $X$ y $Y$ son múltiplos de 5 para todos los rangos.

- Probabilidad del rango (X, Y): Probabilidad de encontrar determinado número de pares ordenados de frecuencias cardiacas en el rango $(X, Y)$ (ecuación 1):

$$
P(X, Y)=\frac{\begin{array}{c}
\text { Numero de pares ordenados } \\
\text { encontrados en el rango } X, Y
\end{array}}{\text { Total de pares ordenados del trazado }}
$$

Entropía $(S)$ del atractor (ecuación 2):

$$
S=-k \sum_{x=1}^{n} \sum_{y-1}^{n} P(X, Y) \times \operatorname{Ln} P(X, Y)
$$

La variable $S$ es la entropía, $P(X, Y)$ es la probabilidad para el rango $X, Y$; $k$ es la constante de Boltzmann, su valor es de $1.38 \times 10^{-23}$ joules/kelvin.

Proporción S/k del atractor (ecuación 3):

$$
\frac{S}{K}=-\sum_{X-1}^{n} \sum_{y-1}^{n} P(X, Y) \times \operatorname{Ln} P(X, Y)
$$

Esta ecuación puede ser reescrita agrupando probabilidades relacionadas con frecuencias de ocupación de acuerdo con valores de unidades $P(U)$, decenas $P(D)$, centenas $P(C)$ y miles $P(M)$. Si se reemplazan estas variables tendremos (ecuación 3a):

$$
\begin{aligned}
& \frac{S}{K} \sum_{u} P(U) \times \operatorname{Ln} P(U)+\sum_{D} P(D) \times \operatorname{Ln} P(D) \\
& +\sum_{C} P(C) \times \operatorname{Ln} P(C)+\sum_{M} P(M) \times \operatorname{Ln} P(M)
\end{aligned}
$$

Que al simplificarla queda de la siguiente forma (ecuación 4):

$$
\frac{S}{K}=U+D+C+M
$$

Donde $\mathrm{U}, \mathrm{D}, \mathrm{C}$ y $\mathrm{M}$ representan la suma de los productos de las probabilidades de los rangos con el logaritmo de esta probabilidad, relacionada con frecuencias de ocupación en unidades, decenas, centenas y miles, respectivamente.

Entendiendo la relación $\mathrm{S} / \mathrm{k}$ como la totalidad, la ecuación se reescribe de esta manera:

$$
T=\frac{S}{K}=U+D+C+M
$$

- Proporciones de la entropía: Proporciones entre las partes y la totalidad definidas como U/T, D/T, $\mathrm{C} / \mathrm{T}, \mathrm{M} / \mathrm{T}, \mathrm{C} / \mathrm{M}$ y $\mathrm{D} / \mathrm{C}$
- Regiones: Zonas específicas dentro del atractor numérico que se establecieron a partir de los prototipos de normalidad y enfermedad de un estudio anterior. ${ }^{16}$ Se determinaron tres regiones: región 1 , todos los rangos de frecuencia cardiaca comunes a todos los registros electrocardiográficos normales; región 2, todos los rangos ocupados por registros normales, menos los de la región 1 ; región 3 , los rangos de frecuencia cardiaca no ocupados por prototipos normales, la región restante dentro del mapa de retardo.

- Rangos de 15 latidos/minuto: Intervalos de frecuencia cardiaca $(\mathrm{FC})$ que incluyen frecuencias agrupadas en múltiplos de 15 latidos/minuto.

- Dimensión fractal estadística: Se desarrolla mediante la linealización logarítmica de la distribución de frecuencias de ocurrencia de los rangos de 15 latidos/minuto (ecuación 5):

$$
D=\frac{\log (\sigma+\mathrm{V})}{\log \left(\frac{F}{P}\right)}
$$

Donde $\mathrm{D}$ es la dimensión estadística, $\mathrm{V}$ es $1 / \mathrm{n}-1$, donde $\mathrm{n}$ es el número de frecuencias obtenido en cada registro, $\mathrm{P}$ es la frecuencia de ocurrencia para cada rango y $\mathrm{F}$ es el factor secundario asociado con el punto de intersección con el eje vertical de la línea obtenida en la linealización.

\section{Población}

Se obtuvieron 10 registros electrocardiográficos y Holter de pacientes mayores de 21 años, cinco con dinámica normal y cinco correspondientes a dinámicas con patología aguda; las dinámicas fueron tomadas de bases de datos del Grupo Insight. El diagnóstico de los registros fue realizado por un cardiólogo experto según los parámetros clínicos convencionales.

\section{Metodología diagnóstica basada en la entropía}

Para cada registro se tomaron los valores máximos y mínimos de la frecuencia cardiaca para cada hora, así como el número de latidos. Con estos valores se simuló una serie de tiempo de la totalidad del registro y posteriormente se generó un atractor numérico ( $\mathrm{Fi}$ gura 1) que cuantifica la frecuencia de ocurrencia para cada rango construido con los valores consecutivos de la frecuencia cardiaca de la serie de tiempo. Se evaluó la probabilidad de ocupación respecto a cada región; se consideró como evento cada rango de frecuencias 


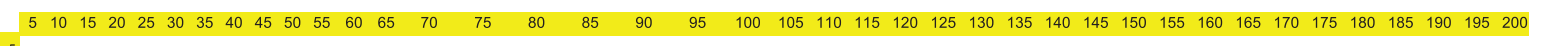

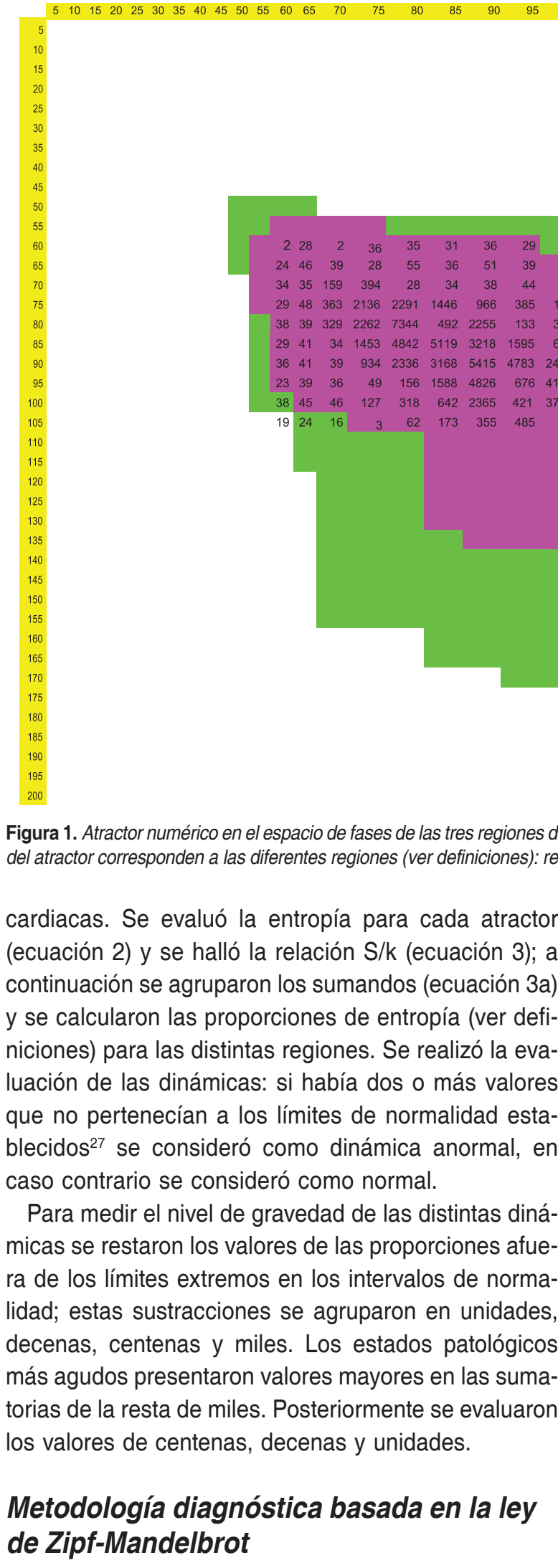

Con los mismos registros se procedió a realizar la aplicación de la ley de Zipf-Mandelbrot. Para ello, las frecuencias cardiacas mínimas y máximas se agruparon en rangos de 15 latidos/minuto y las frecuencias de ocurrencia de cada rango se ordenaron de mayor a menor. Cada rango de frecuencia se asoció con un rango jerárquico cuyos valores oscilaron entre 1 y el número total de rangos presentados en cada dinámica. Estos valores fueron representados en un gráfico, en el que se identificó un comportamiento hiperbólico, luego se hizo una linealización con el fin de encontrar la dimensión fractal estadística (ecuación 5). Las dimensiones fractales para normalidad se encontraron entre 0.70460 y 0.9483 y para enfermedad aguda entre 0.6707 y 0.4228 , de acuerdo con lo establecido previamente por Rodríguez. ${ }^{26}$

\section{Aspectos éticos}

Este estudio fue una investigación de riesgo mínimo, de acuerdo con la resolución 8430 de 1993 del Ministerio de Salud de Colombia, dado que se realizan cálculos físicos y matemáticos con reportes paraclínicos no invasivos anteriormente prescritos según protocolos médicos tradicionales, protegiendo los datos 
e integridad de los participantes. Asimismo, cumple con los principios éticos según la Declaración de HeIsinki de la Asociación Médica Mundial.

\section{Resultados}

\section{Aplicación de la metodología de entropía proporcional}

Los valores de entropía para los registros normales oscilaron entre 6,49E-23 y 7,08E-23, para los registros con patología aguda los valores oscilaron entre 4,64E-23 y 5,06E-23. En los cinco registros normales los valores de las proporciones $\mathrm{S} / \mathrm{k}$ de los atractores correspondientes oscilaron entre -5.1271 y -47033 ; para los cinco pacientes con patología aguda se encontraron entre -3.6648 y -3.3656 . Tanto el valor de entropía como la relación $\mathrm{S} / \mathrm{k}$ diferencian normalidad de enfermedad, con lo que se confirman los resultados previos. ${ }^{27}$

Los valores calculados para proporciones de la entropía de los registros normales se hallaron entre $0 \mathrm{y}$ 0.00838 para $\mathrm{U} / \mathrm{T}$, entre 0 y 0.14056 para $\mathrm{D} / \mathrm{T}$, entre 0 y 0.54730 para $C / T$, entre 0 y 0.45809 para $\mathrm{M} / \mathrm{T}$, entre 1.05529 y 3.22686 para C/M y entre 0.08185 y 0.18142 para $\mathrm{D} / \mathrm{C}$. Se observó que en los pacientes con patología cardiaca aguda los valores de las proporciones de la entropía U/T se hallaron entre 0 y 0.00051 , entre 0 y 0.03570 para $D / T$, entre 0 y 0.24406 para $C / T$, entre 0 y 0.81295 para $M / T$, entre 0 y 1.31495 para C/M y entre 0 y 0.24315 para D/C (Tabla 1 ).

Ningún valor comprendido en la normalidad correspondió a las sumatorias de las restas de las proporciones de entropía fuera de los límites normales (Tabla 1), sin embargo, al menos dos valores para los pacientes con patología aguda se encontraron fuera de estos límites.

Las dinámicas cardiacas con valores más altos en las sumas de las restas de miles corresponden a los estados patológicos con mayor gravedad clínica, los valores menores se relacionan con dinámicas de estados agudos, pero no tan severos como los primeros.

\section{Aplicación de la metodología de Zipf-Mandelbrot}

Se evidenció que el valor de las dimensiones fractales estadísticas de los registros se hallaron entre 0.865 y 0.9892 para normalidad y entre 0.4851 y 0.6067 para las dinámicas patológicas agudas (Tabla 2), con lo que se muestra que para las dinámicas normales se obtuvieron valores de dimensión fractal de hasta más del doble que el valor mínimo de dimensión fractal para las dinámicas con patología aguda; el comportamiento hiperbólico de las dinámicas fue establecido para cada registro.

\section{Discusión}

Este es el primer trabajo en el que se aplica simultáneamente la metodología de entropía proporcional junto con la metodología fundamentada en la ley de Zipf-Mandelbrot. La metodología con sustrato en la entropía proporcional, que requirió la evaluación de los registros electrocardiográficos tanto ambulatorios como continuos y se basó en la probabilidad y proporciones de la entropía, mostró su capacidad predictiva diagnóstica en dinámicas cardiacas y su capacidad de diferenciar adecuadamente dinámicas agudas de dinámicas normales, evidenciando la cuantificación de diferentes niveles de agudización de las dinámicas, así como su aplicación clínica y su utilidad para el seguimiento de pacientes.

Por su parte, la metodología basada en ley de Zipf-Mandelbrot mostró su capacidad de diferenciar patología cardiaca aguda de normalidad en función del grado de complejidad del sistema, más alto en casos normales. Cabe resaltar que ambas metodologías son aplicables a casos particulares independientemente de otras consideraciones; su capacidad diagnóstica es objetiva y reproducible.

La metodología con base en la entropía proporcional ha sido aplicada en una unidad de cuidados intensivos,$^{25}$ donde se observó que al evaluar dinámicas cardiacas por varios días se puede predecir adecuadamente su evolución, lo que probó que el aumento de las proporciones de la entropía es indicativo de agudización de la patología que presenta el paciente. Por otro lado, es menester recordar la aplicabilidad clínica de esta metodología evidenciada en estudios cegados con 450 y 600 casos entre normales y con patología cardiaca. ${ }^{22,23}$ Los resultados de la actual investigación validan los hallazgos anteriores, demostrando la capacidad diagnóstica y predictiva de este método.

Mediante la aplicación de la ley de Zipf-Mandelbrot, Rodríguez ${ }^{28}$ estableció diferencias en el grado de complejidad del repertorio de células $T$ específicas contra el alérgeno Poa $p 9$ en un paciente alérgico ante la presencia y ausencia de $\alpha$ interferón, que mostraron analogía entre los fenómenos del lenguaje ${ }^{11-14}$ y la distribución del repertorio de células T contra el alérgeno. También se aplicó la ley de Zipf-Mandelbrot para desarrollar un nuevo diagnóstico con aplicación clínica 


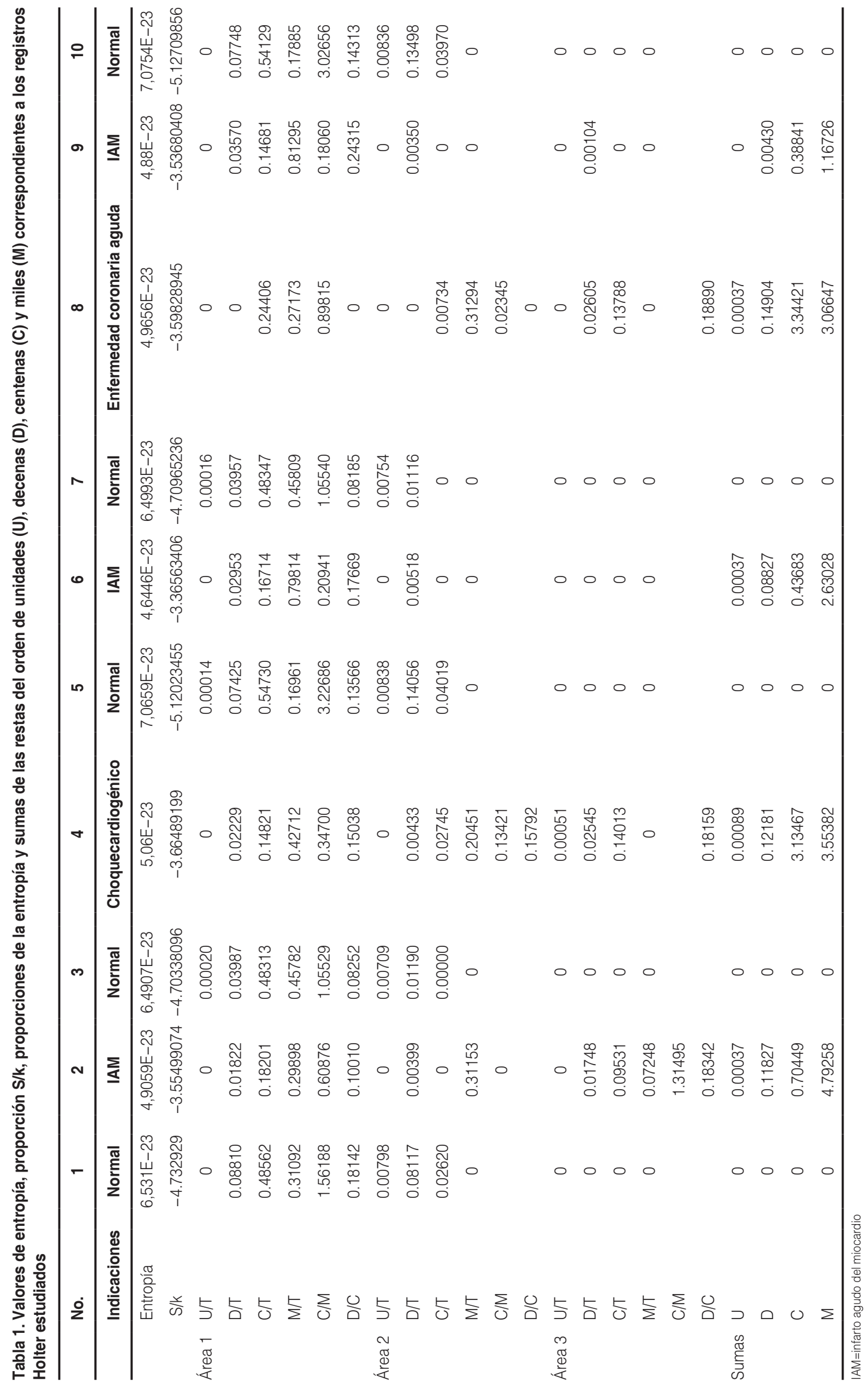


Tabla 2. Valores de la dimensión fractal estadística (DF) y el factor de correlación $\left(R^{2}\right)$ para los Holter estudiados

\begin{tabular}{llcc}
\hline No. & Indicaciones & DF & $\mathbf{R}^{\mathbf{2}}$ \\
\hline 1 & Normal & 0.9406 & 0.7526 \\
2 & Infarto agudo del miocardio & 0.593 & 0.782 \\
3 & Normal & 0.8791 & 0.9523 \\
4 & Choque cardiogénico & 0.4986 & 0.7577 \\
5 & Normal & 0.9892 & 0.9839 \\
6 & Infarto agudo del miocardio & 0.601 & 0.801 \\
7 & Normal & 0.865 & 0.7755 \\
8 & Enfermedad coronaria aguda & 0.4851 & 0.8398 \\
9 & Infarto agudo del miocardio & 0.6067 & 0.9685 \\
10 & Normal & 0.9496 & 0.9774 \\
\hline
\end{tabular}

en la evaluación del monitoreo fetal. ${ }^{29}$ Este método de diagnóstico permite la simplificación de los parámetros clínicos para la evaluación de esta prueba, ya que permite anticipar situaciones adversas en el bienestar fetal con parámetros más objetivos y reproducibles que la evaluación convencional. ${ }^{30,31}$

Se han realizado múltiples trabajos para establecer medidas diagnósticas útiles a través de diferentes tipos de índices, ${ }^{16-18}$ entre los que figuran la entropía de compresión ${ }^{32}$ o DFA (detrended fluctuation analysis), aún sin amplia aplicabilidad clínica. ${ }^{20,33}$ No obstante, la aplicación de la metodología de entropía proporcional ${ }^{23,24}$ no necesita índices pues determina una autoorganización del sistema en términos matemáticos estrictos mediante las proporciones de la entropía, evidenciando el estado general del sistema dinámico cardiaco para cada caso particular.

Se resalta que la aplicación de leyes y teorías físico-matemáticas a los sistemas desde la perspectiva acausal ha sido el sustrato de investigaciones en medicina que han dado lugar a nuevas herramientas diagnósticas y permitido el desarrollo de metodologías predictivas en ámbitos como la infectología, específicamente en la predicción de linfocitos CD4 con parámetros del cuadro hemático, ${ }^{34}$ en inmunología, ${ }^{35}$ biología molecular en temas relevantes como malaria ${ }^{36}$ en salud pública a través de la predicción de brotes de malaria en Colombia, ${ }^{37}$ en estudios a nivel arterial y celular, ${ }^{38,39}$ específicamente en las células del cuello uterino mediante geometría euclidiana y fractal y en las arterias coronarias mediante geometría fractal en un modelo experimental de reestenosis. También se han desarrollado predicciones de mortalidad en una unidad de cuidados intensivos a partir de sistemas dinámicos y teoría deconjuntos ${ }^{40}$ y predicciones de sepsis en la dinámica cardiaca neonatal. ${ }^{41}$

Las metodologías descritas son capaces de caracterizar y predecir dinámicas cardiacas y de otra índole, evidenciando la utilidad práctica de la comprensión fisicomatemática de los fenómenos biomédicos y estableciendo aplicaciones en el aspecto diagnóstico que se traducen en la toma de decisiones oportunas y soluciones aplicables en los diferentes campos de la medicina.

\section{Financiamiento}

Producto derivado del proyecto INV-ING 2096, financiado por la vicerrectoría de Investigaciones de la Universidad Militar Nueva Granada, vigencia 2016.

\section{Agradecimientos}

A la doctora Yanneth Méndez, vicerrectora académica, a la ingeniera Marcela Iregui, vicerrectora de Investigaciones, a la ingeniera Carol E. Arévalo Daza, decana de la Facultad de Ingeniería, y a la doctora Elsa Adriana Cárdenas, directora del Centro de Investigaciones de la Facultad de Ingeniería. A los doctores Tito Tulio Roa, director de Educación Médica, Jorge Ospina, director médico, Alfonso Correa, director del Centro de Investigaciones, y a las doctoras Adriana Lizbeth Ortiz, epidemióloga, Silvia Ortiz, jefa de enfermería del Centro de Investigaciones, y a Sandra Rodríguez, enfermera. También al doctor Fernán Mendoza, cardiólogo de la Fundación Clínica Abood Shaio.

\section{Bibliografía}

1. Devaney RL, Siegel PB, Mallinckrodt AJ, McKay S. A first course in chaotic dynamical systems: theory and experiment. EE. UU.: Addison-Wesley; 1992.

2. Peitgen $\mathrm{H}$. Length area and dimension. Measuring complexity and scalling properties. En: Chaos and fractals: new frontiers of science. EE. UU.: Springer-Verlag; 1992.

3. Goldberger A, Amaral LA, Hausdorff JM, Ivanov PC, Peng CK, Stanley HE. Fractal dynamics in physiology: alterations with disease and aging. Proc Natl Acad Sci USA. 2002; 99(Suppl 1):2466-2472.

4. Laplace Pierre. Ensayo filósofico sobre las probabilidades. Barcelona: Altaya; 1995.

5. Matvéev A. Física molecular. Moscú: Mir; 1987.

6. Tolman R. Principles of statistical mechanics. EE. UU.: Dover Publications; 1979

7. Feynman RN, Leighton RB, Sands M. Leyes de la termodinámica. En: Física. Volumen 1. EE. UU.: Addison-Wesley; 1964.

8. Pincus SM, Gladstone IM, Ehrenkranz RA. A regularity statistic for medical data analysis. J Clinmonit. 1991;7:335-45.

9. Guillén $P$, Vallverdú M, Rojas R, Jugo D, Carrasco H, Caminal P. Dinámica no lineal para caracterizar la variabilidad del ritmo cardiaco en pacientes chagásticos. En: Memorias del II Congreso Latinoamericano de Ingeniería Biomédica, Cuba, 2001. Ciencia. 2003;11(4):276-283.G

10. Vikman S, Mäkikallio TH, Yli-Mäyry S, Pikkujämsä S, Koivisto AM, Reinikainen $\mathrm{P}$, et al. Altered complexity and correlation properties of $\mathrm{R}-\mathrm{R}$ interval dynamics before the spontaneous onset of paroxysmal atrial fibrillation. Circulation. 1999;100:2079-2084. 
11. Zipf G. Human behavior and the principle of least effort: an introduction to human ecology. EE. UU.: Addison-Wesley; 1949.

12. Mandelbrot BB. Scaling and power laws without geometry. En: The fractal geometry of nature. EE. UU.G: Freeman; 1972.

13. Mandelbrot BB. Árboles jerárquicos o de clasificación, y la dimensión. En: Los objetos fractales. Barcelona: Tusquets; 2000.

14. Mandelbrot B. Structure formelle des textes et comunication. World 1954:10(1):1-27.

15. Organización Mundial de la Salud. Enfermedades cardiovasculares. Ginebra, Suiza: OMS; 2011.

16. Norris PR, Anderson SM, Jenkins JM, Williams AE, Morris JA. Heart rate multiscale entropy at three hours predicts hospital mortality in 3,154 trauma patients. Shock. 2008;30:17-22.

17. Huikuri HV, Mäkikallio TH, Peng CK, Goldberger AL, Hintze U, Moller M Fractal correlation properties of R-R interval dynamics and mortality in patients with depressed left ventricular function after an acute myocardial infarction. Circulation. 2000;101:47-53.

18. Mäkikallio TH, Hoiber S, Kober L, Torp-Pedersen C, Peng CK, Goldberger $\mathrm{AL}$, et al. Fractal analysis of heart rate dynamics as a predictor of mortality in patients with depressed left ventricular function after acute myocardial infarction. TRACE Investigators. TRAndolapril Cardiac Evaluation. Am J Cardiol. 1999;83:836-839.

19. Rodríguez J, Correa C, Melo M, Domínguez D, Prieto S, Cardona DM, et al. Chaotic cardiac law: developing predictions of clinical application. J Med Sci. 2013;4(2):79-84

20. Perkiömäki JS, Mäkikallio TH, Huikuri HV. Fractal and complexity measures of heart rate variability. Clin Exp Hypertens. 2005;27:149-158.

21. Buccelletti F, Bocci MG, Gilardi E, Fiore V, Calcinaro S, Fragnoli C, et al Linear and nonlinear heart rate variability indexes in clinical practice. Comput Math Methods Med. 2012;2012:219080.

22. Rodríguez J, Prieto S, Domínguez D, Melo M, Mendoza F, Correa C, et al. Mathematical-physical prediction of cardiac dynamics using the proportional entropy of dynamic systems. J Med Med Sci. 2013; 4(8):370-381.

23. Rodríguez J, Prieto S, Correa C, Soracipa Y, Aguirre G, Méndez L. Proportional entropy applied to the clinical diagnostic of cardiac dynamic: blind study with 600 holter. En: Memorias The $61^{\text {st }}$ Annual Conference of the Israel Heart Society in association with The Israel Society of Cardiothoracic Surgery, 2014.

24. Rodríguez J, Prieto S, Bernal P, Izasa D, Salazar G, Correa C, et al. Entropía proporcional aplicada a la evolución de la dinámica cardiaca. Predicciones de aplicación clínica. En: Rodríguez LG, coordinador. La emergencia de los enfoques de la complejidad en América Latina: implicancias políticas, epistemológicas y metodológicas para las ciencias del siglo XXI. Tomo 1. Buenos Aires: Comunidad Editora Latinoamericana 2015.

25. Rodríguez J. Mathematics physical assessment of cardiac dynamics based on theory of probability and proportions of entropy in the intensive care unit for patients with arrhythmia. Reino Unido: Medical Physics; 2015.
26. Rodríguez J, Prieto S, Correa C, Mendoza F, Weiz G, Soracipa Y, et al. Physical mathematical evaluation of the cardiac dynamic applying the Zipf-Mandelbrot law. JMP. 2015;6:1881-1888.

27. Rodríguez J. Entropía proporcional de los sistemas dinámicos cardiacos. Predicciones físicas y matemáticas de la dinámica cardiaca de aplicación clínica. Rev Col Cardiol. 2010;17(3):115-129.

28. Rodríguez J. Comportamiento fractal del repertorio $T$ específico contra el alergeno Poa p9. Rev Fac Med Univ Nac Colomb. 2005;53:72-78.

29. Rodríguez J, Prieto S, Ortíz L, Bautista A, Bernal P, Avilán N. Diagnóstico matemático de la monitoria fetal aplicando la ley de Zipf-Mandelbrot. Rev Fac Med Univ Nac Colomb. 2006;54(2): 96-107.

30. Borgatta L, Shrout PE, Divon MY. Reliability and reproducibility of nonstress test readings. Am J Obstet Gynecol. 1988;159(3):554-558.

31. Rodríguez J. Dynamical systems theory and Zipf-Mandelbrot law applied to the development of a fetal monitoring diagnostic methodology. En: Memorias del XVIII FIGO World Congress of Gynecology and Obstetrics, Kuala lumpur, Malaysia, 2006.

32. Baumert M, Baier V, Haueisen J, Wessel N, Meyerfeldt U, Schirdewan A et al. Forecasting of life threatening arrhythmias using the compression entropy of heart rate. Methods Inf Med. 2004;43:202-206.

33. Voss A, Schulz S, Schroeder R, Baumert M, Caminal P. Methods derived from nonlinear dynamics for analysing heart rate variability. Phil Trans A Math Phys Eng Sci. 2009;367:277-296.

34. Rodríguez J, Prieto S, Correa C, Pérez C, Mora J, Bravo J, et al. Predictions of CD4 lymphocytes' count in HIV patients from complete blood count. BMC Med Phys. 2013;13:3.

35. Rodríguez J. Teoría de unión al HLA clase II: teoría de probabilidad, combinatoria y entropía aplicadas a secuencias peptídicas. Inmunologia. 2008;27(4):151-166.

36. Rodríguez J, Bernal P, Prieto S, Correa C. Teoría de péptidos de alta unión de malaria al glóbulo rojo. Predicciones teóricas de nuevos péptidos de unión y mutaciones teóricas predictivas de aminoácidos críticos. Inmunologia. 2010;29(1):7-19.

37. Rodríguez J. Método para la predicción de la dinámica temporal de la malaria en los municipios de Colombia. Rev Panam Salud Pública. 2010;27(3):211-218.

38. Rodríguez J, Prieto S, Correa C, Bernal P, Puerta G, Vitery S, et al. Theoretical generalization of normal and sick coronary arteries with fractal dimensions and the arterial intrinsic mathematical harmony. BMC Med Phys. 2010;10:1.

39. Prieto S, Rodríguez J, Correa C, Soracipa Y. Diagnosis of cervical cells based on fractal and euclidian geometrical measurements: intrinsic geometric cellular organization. BMC Med Phys. 2014;14:2.

40. Rodríguez J. Dynamical systems applied to dynamic variables of patients from the intensive care unit (ICU): physical and mathematical mortality predictions on ICU. J Med Sci. 2015;6(8):102-108.

41. Rodríguez J, Prieto S, Flórez M, Alarcón C, López R, Aguirre G, et al. Physical-mathematical diagnosis of cardiac dynamic on neonatal sepsis: predictions of clinical application. J Med Sci. 2014;5(5):102-108. 